\title{
Detailed $f_{1}, f_{2}$ Area Study of Distortion Product Otoacoustic Emissions in the Frog
}

\author{
Sebastiaan W. F. Meenderink, ${ }^{1,2}$ Peter M. Narins, ${ }^{3}$ and Pim van Dijk ${ }^{4,5}$ \\ ${ }^{1}$ Department of Otorhinolaryngology and Head and Neck Surgery, University Hospital Maastricht, 5800, 6202 AZ, Maastricht, \\ The Netherlands \\ ${ }^{2}$ Institute for Brain and Behaviour, Maastricht University, Maastricht, The Netherlands \\ ${ }^{3}$ Department of Physiological Science, University of California, Los Angeles, Los Angeles, CA 90095-1606, USA \\ ${ }^{4}$ Department of Otorhinolaryngology, University Medical Centre, Groningen, The Netherlands \\ ${ }^{5}$ School of Behavioral and Cognitive Neurosciences, Groningen, The Netherlands
}

Received: 11 March 2004; Accepted: 12 October 2004; Online publication: 2 February 2005

\begin{abstract}
Distortion product otoacoustic emissions (DPOAEs) are weak sounds emitted from the ear when it is stimulated with two tones. They are a manifestation of the nonlinear mechanics of the inner ear. As such, they provide a noninvasive tool for the study of the inner ear mechanics involved in the transduction of sound into nerve fiber activity. Based on the DPOAE phase behavior as a function of frequency, it is currently believed that mammalian DPOAEs are the combination of two components, each generated by a different mechanism located at a different location in the cochlea. In frogs, instead of a cochlea, two separate hearing papillae are present. Of these, the basilar papilla (BP) is a relatively simple structure that essentially functions as a single auditory filter. A two-mechanism model of DPOAE generation is not expected to apply to the BP. In contrast, the other hearing organ, the amphibian papilla (AP), exhibits a tonotopic organization. In the past it has been suggested that this papilla supports a traveling wave in its tectorial membrane. Therefore, a two-mechanism model of DPOAE generation may be applicable for DPOAEs from the AP. In the present study we report on the amplitude and phase of DPOAEs in
\end{abstract}

Correspondence to: Sebastiaan W. F. MeenderinkDepartment of Otorhinolaryngology and Head and Neck Surgery - University Hospital Maastricht - P.O. Box 5800, 6202 AZ Maastricht, The Netherlands. Telephone: (31) 43-3877593; fax: (31) 43-3877598; email: b.meenderink@kno.unimaas.nl the frog ear in a detailed $f_{1}, f_{2}$ area study. The result is markedly different from that in the mammalian cochlea. It indicates that DPOAEs generated by neither papilla agree with the two-mechanism traveling wave model. This confirms our expectation for the $\mathrm{BP}$ and does not support the hypothesized presence of a mechanical traveling wave in the AP.

Keywords: distortion product otoacoustic emissions, amphibian, frog, traveling wave, two-mechanism DPOAE model

Abbreviations: $\mathrm{AP}$-amphibian papilla; $\mathrm{BP}$ - basilar papilla; DPOAE-distortion product otoacoustic emission

\section{INTRODUCTION}

Distortion product otoacoustic emissions (DPOAEs) are an acoustic phenomenon that can be observed in a healthy ear that is stimulated with two stimulus tones with properly chosen frequencies $\left(f_{1}, f_{2}\right.$, with $\left.f_{1}<f_{2}\right)$ and levels $\left(L_{1}\right.$ and $L_{2}$, respectively). It is currently believed that in the mammalian cochlea, lowersideband DPOAEs (with $f_{d p}<f_{1}, f_{2}$ ) are the result of two DPOAE components, each originating from a different location on the basilar membrane (Kim 1980; Kemp and Brown 1983; Brown et al. 1996; Talmadge et al. 1999; Shera and Guinan 1999).

Shera and Guinan (1999) postulate that the fundamental distinction between these two compo- 
nents is not the different location but the different mechanism involved in their generation. Hence, they use the term two-mechanism model of DPOAE generation. Here, nonlinear distortion generates the initial DPOAE component (at the overlap region of the two stimulus tones) that may travel both in basal and apical directions in the cochlea. The apically traveling DPOAE energy may be reflected because of local irregularities in the fine structure of the basilar membrane (around the characteristic frequency place of the distortion product) resulting in a second DPOAE component traveling to the base of the cochlea. The combination of the two basally traveling components, together with multiple internal reflections (Stover et al. 1996), results in the DPOAE that can be recorded in the ear canal.

The frog ear is an interesting model for studying DPOAEs because, instead of a cochlea, it contains two distinct papillae that respond to airborne sound. The amphibian papilla (AP) consists of an elongated strip of sensory epithelium. It exhibits tonotopic organization (Lewis et al. 1982), and the presence of traveling waves in the tectorial membrane has been proposed (Lewis and Leverenz 1983; Hillery and Narins 1984; Lewis 1984). Hair cells are innervated by both afferent and efferent nerve fibers, the former having tuning characteristics similar to those found in mammals (Lewis 1992). The AP may generate both spontaneous and distortion product OAEs (Van Dijk and Manley 2001). The analogy of this papilla with the mammalian cochlea, i.e., the presence of tonotopic organization, suggests that a traveling wave mechanism, with separate emission sources, may underlie DPOAE generation.

The basilar papilla (BP) is a much simpler structure. It consists of a small patch of sensory epithelium which is covered by a tectorial membrane (Lewis and Narins 1999). In Rana catesbeiana, hair cells in the BP are only innervated by afferent nerve fibers (Robbins et al. 1967; Frishkopf and Flock 1974). In individual frogs, the vast majority of nerve fibers is tuned to a single frequency (Ronken 1991; Van Dijk et al. 1997). Also, in ranid frogs the hair bundles are oriented in parallel (Lewis 1978). These properties indicate that this papilla functions essentially as a single auditory filter (Ronken 1990; Van Dijk and Manley 2001), which makes it unlikely that DPOAE generation involves two different emission sources or a traveling wave mechanism.

Recently, Knight and Kemp (2000) reported an $f_{1}$, $f_{2}$ area study of human DPOAEs. They showed that patterns in the amplitude and phase data, when represented in an $f_{2} / f_{1}$ versus $f_{d p}$ plot, are oriented either horizontally or vertically. The patterns are consistent with the two-mechanism model of DPOAE generation. A similar method to analyze the phase data was presented by Schneider et al. (2003). Although these methods offer no technique to study the two components separately, they do provide a way to study which component dominates the recorded DPOAE.

In the present study, we report an $f_{1}, f_{2}$ area study of amplitude and phase of DPOAEs in the leopard frog, Rana pipiens pipiens. The patterns observed deviate considerably from those observed in mammals. We conclude that a cochlear-like, two-source DPOAE model does not apply to the amphibian inner ear. This confirms our expectations for the BP, but does not support the hypothesized presence of a mechanical traveling wave in the AP.

\section{METHODS}

Distortion product otoacoustic emissions were recorded from Northern leopard frogs (Rana pipiens pipiens) $: n=5$ females, body mass $=20.8-31.9 \mathrm{~g}$ $($ mean, $27.2 \mathrm{~g})$, snout-vent length $=7.11-7.84 \mathrm{~cm}$ (mean, $7.42 \mathrm{~cm}$ ). Animals were anesthetized with an intramuscular injection of pentobarbital sodium solution (Nembutal, $60 \mathrm{mg} / \mathrm{ml} ; 1.0 \mu \mathrm{l} / \mathrm{g}$ body mass) in one of the hindlimbs. Measurements were performed in a sound-attenuating chamber, with the frog placed on a vibration isolation table. During the experiments, the animal was covered by gauze soaked in tap water to prevent dehydration and to facilitate cutaneous respiration. In each subject the left ear was tested.

DPOAEs were recorded with a probe assembly that contained two miniature transducers (ER-10C; Etymotic Research) for stimulus generation, and one 1/2-in. condenser microphone (Brüel and Kjær type 4134) for emission recording. The open end of the probe assembly was carefully placed against the skin surrounding the frog's tympanic membrane. A tight seal between probe and skin was obtained by using silicone grease.

DPOAEs were evoked by two stimulus tones, with frequencies $f_{1}$ and $f_{2}$ (where $f_{2} / f_{1}>1$ ) and levels $L_{1}$ and $L_{2}$, each of which was played from a separate miniature speaker. The stimulus tones were generated from two separate D/A channels (RP2; Tucker Davis Technologies, Gainesville, FL, USA) and the level of each tone was adjusted with a separate programmable attenuator (PA5: Tucker Davis Technologies). The microphone signal was amplified 60 dB with a preamplifier (Brüel and Kjær type 2609) and recorded on computer disk using an A/D converter (RP2; Tucker Davis Technologies). During the experiments, the amplified microphone signal was also fed into a spectrum analyzer (Stanford 
Research Systems SR770) in order to monitor the DPOAEs online. Customized software, written in Matlab (The MathWorks, Inc.) and RPvds (Tucker Davis Technologies) were used to control stimulus tone generation and signal recording.

For each frog, recordings were made in a series of fixed-frequency ratio (fixed- $f_{2} / f_{1}$ ) sweeps from low to high $f_{1}$, starting with the lowest frequency ratio $f_{2} / f_{1}=$ 1.02 and ending with a ratio of 1.70 . In each sweep, $f_{1}$ varied from 213 to $2774 \mathrm{~Hz}$ in approximately $30 \mathrm{~Hz}$ steps. The levels of the stimulus tones, $L_{1}=L_{2}=76$ dB SPL, were kept constant for all stimulus frequency combinations. These relatively large stimulus amplitudes were used in order to evoke DPOAEs over a wide enough stimulus frequency $\left(f_{1}, f_{2} / f_{1}\right)$ area to reliably unwrap the phase data. At the start and at regular intervals during the experiment, a calibration procedure was performed to set the levels of the stimulus tones. In addition, the condition and the position of the animal were checked, and the gauze covering the animal remoisturized. To determine the nonlinear distortion of the DPOAE recording setup, experiments were performed with the open end of the probe pressed against a solid surface. This resulted in no detectable system distortion for any of the stimulus conditions used.

At each frequency pair $\left(f_{1}, f_{2}\right)$, stimulus tones were played continuously for 50,000 sample points $(\approx 4.10 \mathrm{~s}$ with a sample frequency of $12.2 \mathrm{kHz})$. Frequency $f_{1}$ was chosen such that 100 periods of this stimulus tone matched exactly an integer number of sample points. By performing the experiments in the form of fixed-ratio sweeps, where the ratio $f_{2} / f_{1}$ was chosen with a maximum of two significant digits (e.g., $f_{2} / f_{1}=1.04$ or $f_{2} / f_{1}=1.30$ ), all other frequencies of interest (i.e., stimulus frequency $f_{2}$ and all DPOAEs) were also exactly periodic over the same integer number of sample points. This method has two advantages in the analysis of the recorded signal. First, the total recorded signal (50,000 sample points) can be divided into a series of blocks that all have the same starting phase of the stimulus tones and DPOAEs. These blocks can be averaged in order to reduce the noise floor, without affecting the amplitude and phase of the tones of interest. Second, the exact periodicity of all tones of interest ensures that in the Fourier analysis the corresponding frequencies each fall exactly in the center of a frequency bin. This abolishes spectral smearing, and provides an accurate estimate of the amplitudes and phases of the DPOAEs.

In the analysis of the digitized microphone signal, the first periodic block was omitted to exclude onset phenomena. The remaining signal was used to calculate the amplitudes $\left(L_{1}, L_{2}\right)$ and phases $\left(\phi_{f_{1}}\right.$, $\phi_{f_{2}}$ ) of the stimulus tones using Fourier analysis. The parameters thus obtained were used to subtract the stimulus tones from the microphone signal in the time domain. For the remaining signal, containing only the DPOAEs and system noise, each periodic block was subjected to a level-crossing artifact rejection method. Artifact-free blocks were subaveraged in two buffers, $A$ and $B$. The average $(A+B) / 2$ was used to estimate the DPOAEs' amplitudes and phases, whereas the difference $A-B$ provided an estimate for the noise levels. As with the stimulus tones, the amplitudes and phases of the DPOAEs were calculated using Fourier analysis.

The "Principles of Animal Care" (NIH publication 85-23, revised 1985) and US regulations were followed throughout this study, and the protocols were approved by the University of California Animal Research Committee.

\section{Data analysis and representation}

For each distortion product, amplitude and phase data were arranged in an $\left(f_{1}, f_{2} / f_{1}\right)$ area matrix. Only data points for which the emission amplitude exceeded the noise floor by $6 \mathrm{~dB}$ were included in the matrices. Phase data are represented relative to the phase of the stimulus tones. That is, for the distortion product at $f_{d p}=(n+1) f_{1}-n f_{2}$, the relative phase is: $\Phi_{f_{d p}}=\phi_{f_{d p}}-(n+1) \phi_{f_{1}}+n \phi_{f_{2}}$. Subsequently, the phase data were unwrapped in two dimensions (constant $f_{1}$ and constant $f_{2} / f_{1}$ ) by removing $2 \pi$ discontinuities.

Phase slope delay (group delay) is defined as:

$$
D=-\frac{1}{2 \pi} \frac{d \Phi_{d p}}{d f_{d p}}
$$

where $\Phi_{d p}$ is the relative phase of the distortion product and is considered as a function of $f_{1}$ for a constant ratio $f_{2} / f_{1}$. The slope $d \Phi / d f$ of the phase was calculated by fitting a straight line to three points, $f_{1}$ and the two neighboring points. This was only performed when all three points had a DPOAE amplitude exceeding the noise floor by $6 \mathrm{~dB}$. To prevent contamination of the results, only slopes with a correlation coefficient exceeding 0.988 (corresponding to $p=0.10$ ) are considered in this paper.

\section{RESULTS}

Distortion product otoacoustic emissions could be detected in each individual frog investigated. We systematically analyzed the recorded data for distortion products at frequencies $2 f_{1}-f_{2}, 2 f_{2}-f_{1}, 3 f_{1}-$ $2 f_{2}$, and $3 f_{2}-2 f_{1}$.

The dependence of DPOAE amplitude on the stimulus frequencies follows a complicated pattern. 
a)
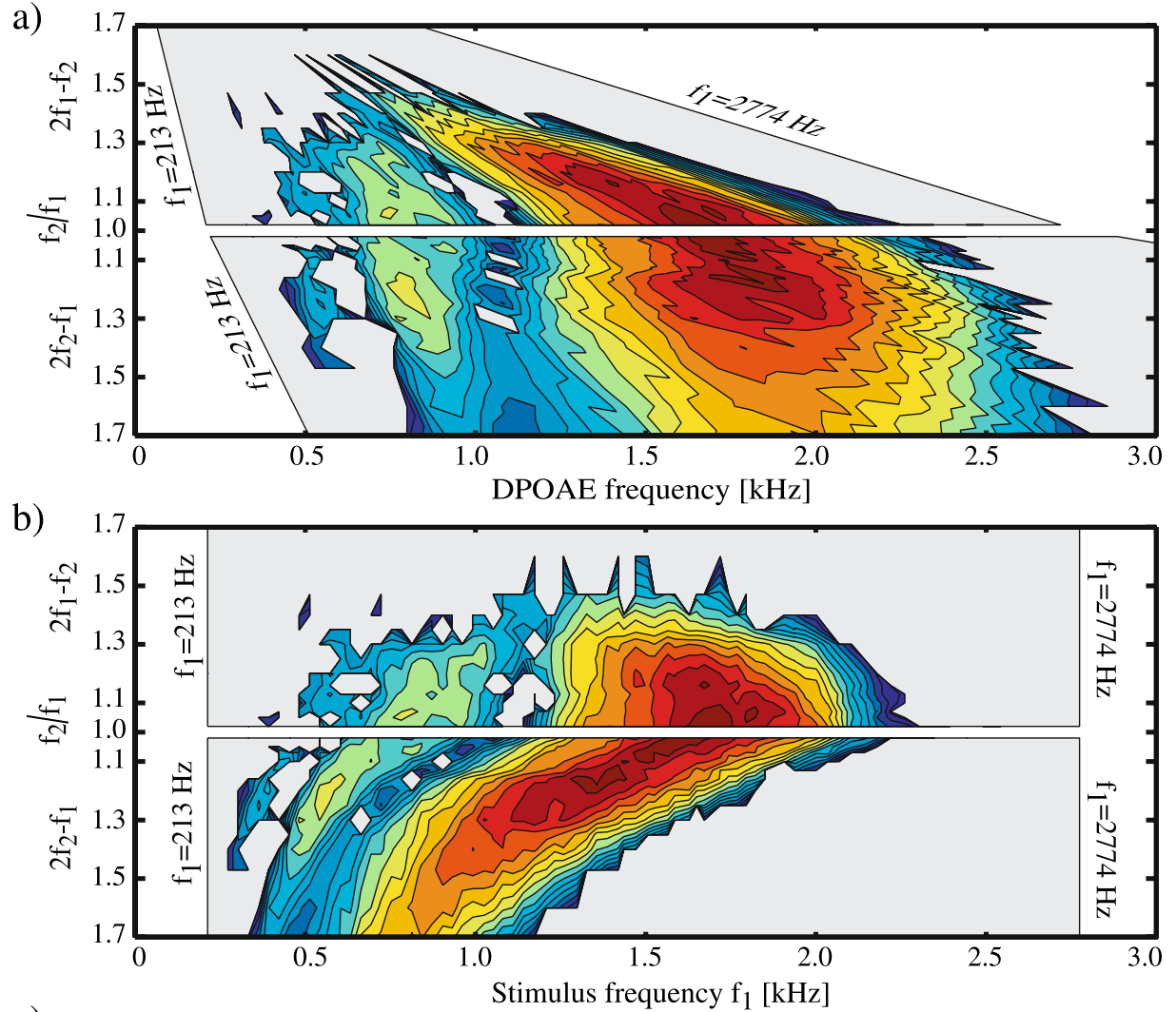

c)

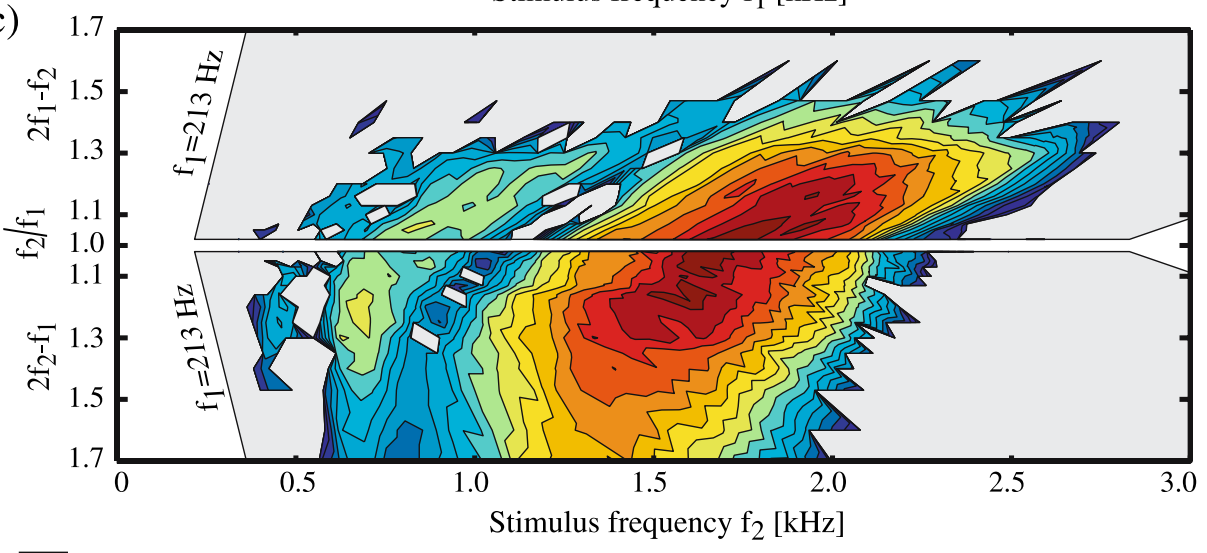

$\begin{array}{lll}: 0.22 & \square: 6.8 & \square: 12 . .14 \\ : 2.4 & \square: 8.10 & \square: 14 . .16\end{array}$

$: 4.6 \square: 10 . .12$

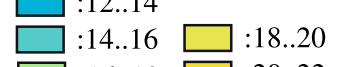

$: 16 . .18$

$: 20 . .22$

$: 22 . .24$

24.26

$: 26 . .28$

$28 . .30$

$: 30 . .32$

$: 32 . .34$

FIG. 1. Amplitude at $2 f_{1}-f_{2}$ and $2 f_{2}-f_{1}$ in $\mathrm{dB} S \mathrm{SPL}$, evoked with $L_{1}=L_{2}=76 \mathrm{~dB} \mathrm{SPL}$, recorded in one frog. The different panels represent the same data set with $f_{2} / f_{1}$ versus either (a) distortion product frequency, (b) stimulus frequency $f_{1}$, or (c) stimulus frequency $f_{2}$. In each panel, the same color-coding is used. Individual contour lines are drawn at 2-dB intervals. The shaded area represents the $\left(f_{1}, f_{2}\right)$ area studied. For DPOAEs at $2 f_{1}-f_{2}$, amplitude peaks and valleys result in patterns that are vertical when data are plotted as function of primary frequency $f_{1}$ (panel $\mathbf{b}$, upper half). In contrast, for DPOAE at $2 f_{2}-f_{1}$, a similar vertical pattern occurs when the data are plotted as a function of either primary frequency $f_{2}$ (panel c, lower half) or DPOAE frequency (panel a, lower half). Note that two broad frequency regions can be seen where emission amplitudes show a relative maximum. These two regions are separated by a notch region centered around $f_{1}=1250 \mathrm{~Hz}$ (for $2 f_{1}$ $-f_{2}$ ) or $f_{\mathrm{dp}}$ slightly below $1250 \mathrm{~Hz}$ (for $2 f_{2}-f_{1}$ ), where emissions are considerably lower in amplitude or even undetectable. This frequency of $1250 \mathrm{~Hz}$ corresponds with the separation in characteristic frequency ranges of nerve fibers from the amphibian and basilar papilla (Ronken 1991).
Some of these patterns are illustrated by the contour lines in Figure 1. In each panel of Figure 1, the bottom half refers to the DPOAE at $2 f_{2}-f_{1}$, and the top half illustrates DPOAE at $2 f_{1}-f_{2}$. In general, DPOAE amplitudes were relatively large in two broad frequency ranges. Separating these two peak regions is a well-defined valley where DPOAE amplitudes dropped considerably. As is obvious from the figure, the orientation of these DPOAE peak and valley regions depends on the parameter plotted on the abscissa. When the DPOAE at $2 f_{1}-f_{2}$ is plotted as a function of stimulus frequency $f_{1}$
(Fig. 1b, upper half), these DPOAE amplitude peaks and valleys give rise to a vertical pattern in the contour lines. In contrast, when the amplitude of this DPOAE is plotted as a function of either DPOAE frequency (Fig. 1a, upper half) or stimulus frequency $f_{2}$ (Fig. 1c, upper half), a diagonal pattern in the contour lines appears.

Inspection of the lower halves of the panels in Figure 1 makes it clear that for the DPOAE at $2 f_{2}-f_{1}$ the situation is similar, but slightly more complex. Here, the peaks and valleys in DPOAE amplitude result in an approximately vertical pattern in the 


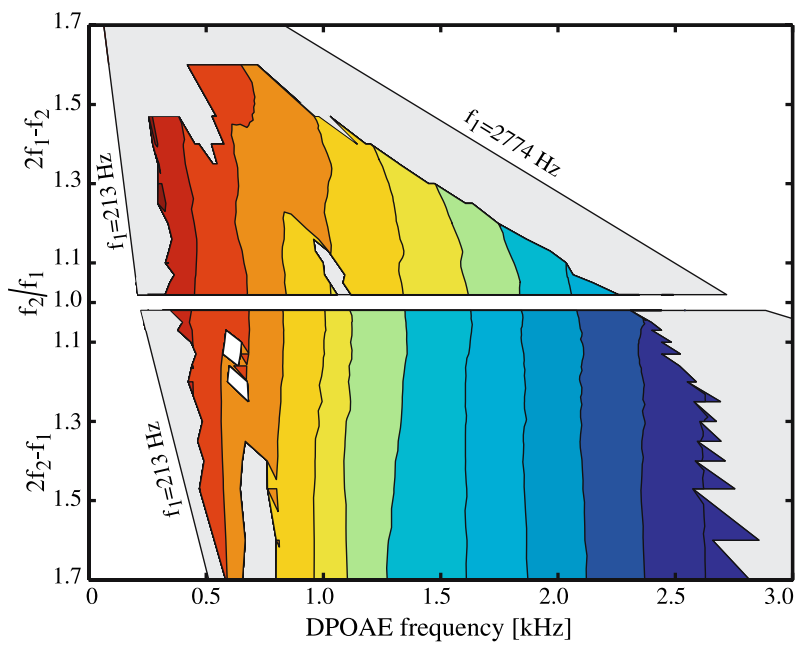

FIG. 2. Phase data (in rad) for $2 f_{1}-f_{2}$ and $2 f_{2}-f_{1}$ plotted as a function of distortion product frequency. The phase data correspond to the amplitude data represented in Figure 1. Contour lines are drawn at $\pi$ rad intervals. The shaded area represents the $\left(f_{1}, f_{2}\right)$ area studied. The vertical contour lines indicate that the relative phase of the DPOAEs depends on the frequency of the distortion product itself, and is independent of both the absolute and relative frequencies of the two stimulus tones.

contour plots when either the DPOAE frequency (Fig. 1a) or the stimulus frequency $f_{2}$ (Fig. 1c) is on the abscissa. With the stimulus frequency $f_{1}$ on the horizontal axis (Fig. 1b), the pattern in the contour plot is diagonal.

DPOAEs at $2 f_{2}-f_{1}$ could be detected for much wider stimulus frequency ratios than those at $2 f_{1}-f_{2}$. For example, at the largest ratio studied $\left(f_{2} / f_{1}=1.7\right.$; i.e., the horizontal boundaries of the panels), DPOAEs at $2 f_{2}-f_{1}$ were still as large as $26 \mathrm{~dB}$ SPL, while for the same ratio DPOAEs at $2 f_{1}-f_{2}$ were indistinguishable from the noise floor.

DPOAE audiograms recorded in the frog typically show a bimodal dependence on frequency (Van Dijk and Manley 2001; Van Dijk et al. 2003; Meenderink and Van Dijk 2004). They exhibit two frequency regions with elevated DPOAE amplitudes which are separated by a clear notch in DPOAE amplitude. The area representations in Figure 1 show that this bimodal dependence is observed irrespective of the stimulus frequency ratio $f_{2} / f_{1}$ used. At the same time, these representations show that whether the notch shifts in frequency for DPOAE audiograms recorded with different frequency ratios $f_{2} / f_{1}$ depends on the parameter plotted on the $x$-axis. For DPOAEs at $2 f_{1}-f_{2}$, the notch in DPOAE audiograms does not shift position when stimulus frequency $f_{1}$ is on the $x$-axis-it is centered around approximately $f_{1}=1250$ Hz. For DPOAEs at $2 f_{2}-f_{1}$ the notch is observed at a fixed frequency slightly below $f_{d p}=1250 \mathrm{~Hz}$ when distortion product frequency is on the abscissa.
Compared to the amplitude, the pattern observed in the DPOAE phase is simpler. Figure 2 illustrates the phase data accompanying the amplitude data presented in Figure 1. Again, the bottom half of the panel illustrates DPOAEs at $2 f_{2}-f_{1}$, and the top half refers to DPOAEs at $2 f_{1}-f_{2}$. For DPOAEs at both $2 f_{1}-f_{2}$ and $2 f_{2}-f_{1}$, contour lines, i.e., lines of equal relative phase of the DPOAEs, are (nearly) vertical when plotted as a function of DPOAE frequency (Fig. 2). These vertical contour lines indicate that DPOAE phase primarily depends on DPOAE frequency, and is nearly independent of the stimulus frequency ratio $f_{2} / f_{1}$.

The distance between the contour lines in the phase data showed a bimodal dependence on DPOAE frequency, similar to the bimodal dependence observed in the DPOAE amplitude. This is reflected in the group delays of the DPOAEs. Figure 3 a shows the combined group delays (average
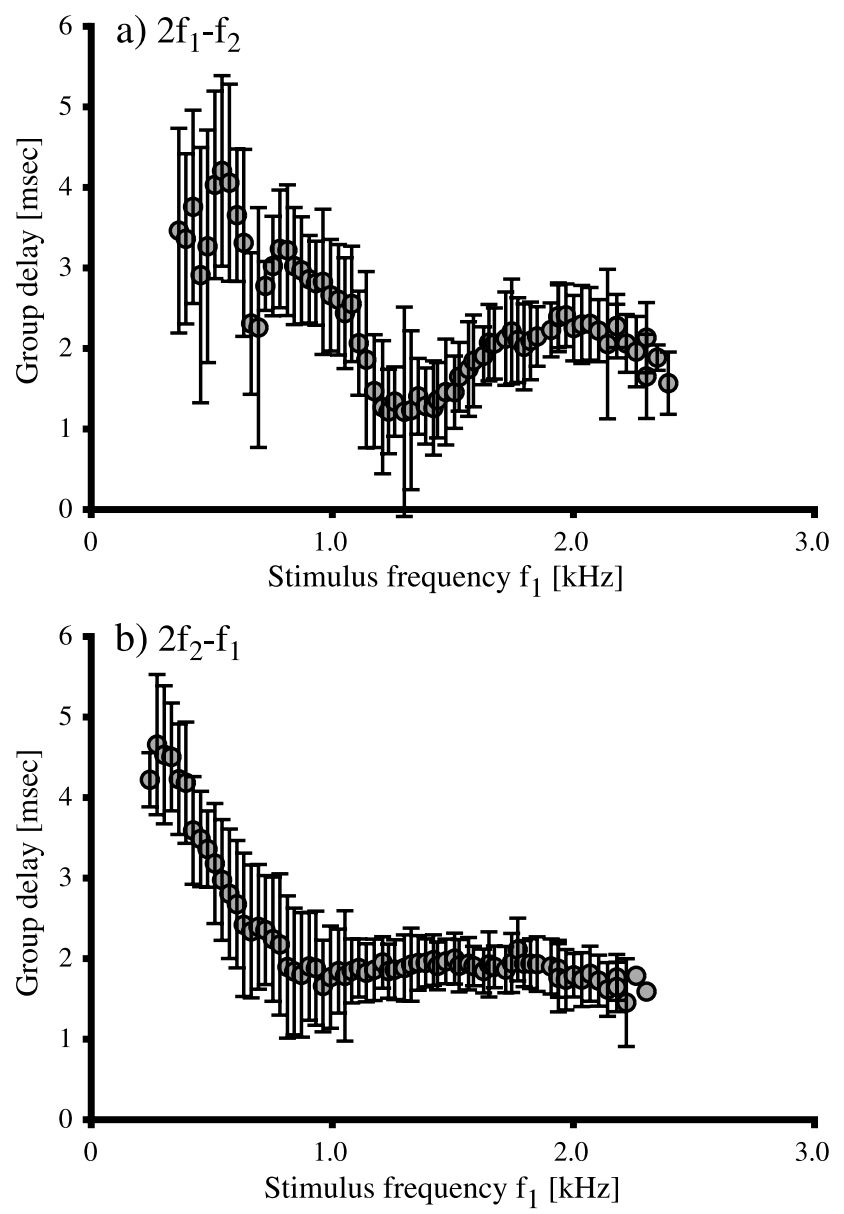

FIG. 3. Group delays calculated for a fixed-ratio recording paradigm plotted as a function of primary frequency $f_{1}$. The two panels represent group delays for (a) $2 f_{1}-f_{2}$ and (b) $2 f_{2}-f_{1}$. Each point gives the combined average over all frogs and all $f_{2} / f_{1}$ ratios. The error bars denote the standard deviation ( \pm 1 SD). Points lacking an error bar represent single observations. 

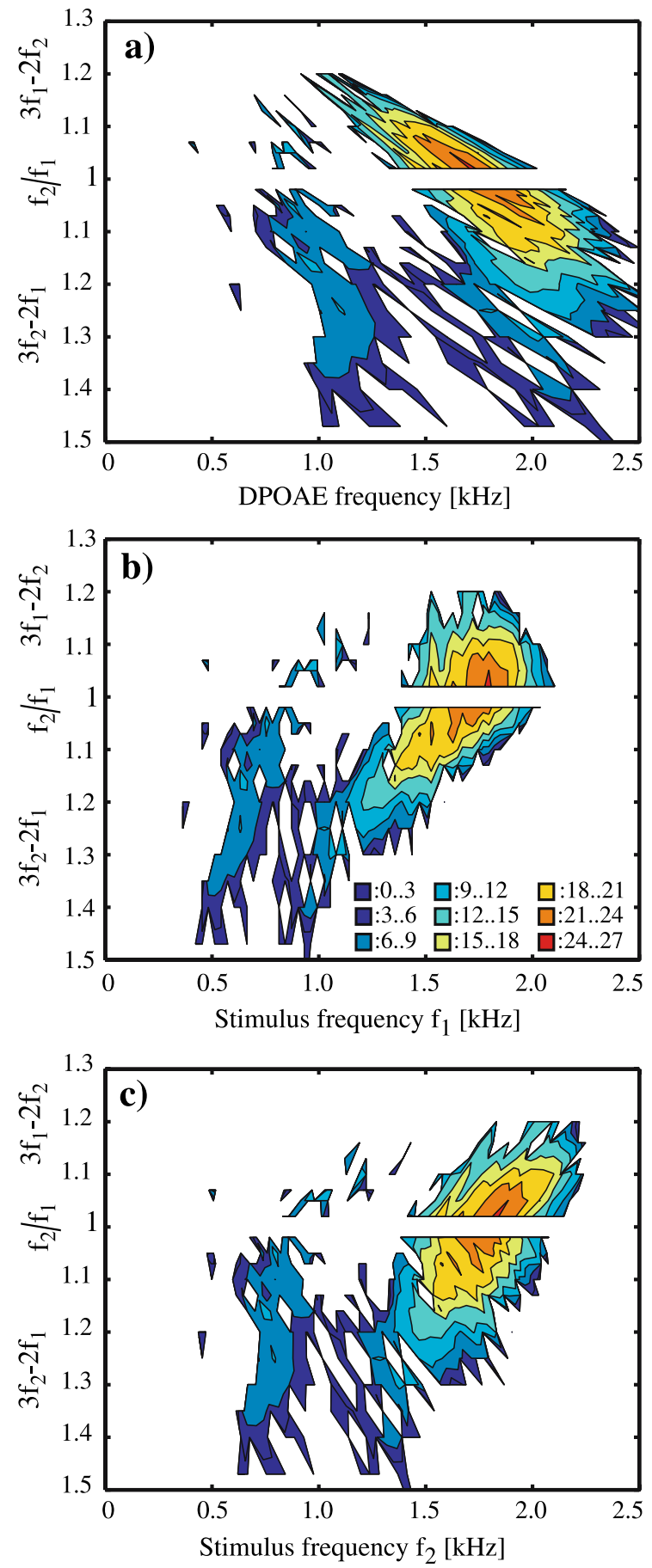

FIG. 4. Amplitude of DPOAEs at $3 f_{1}-2 f_{2}$ and $3 f_{2}-2 f_{1}$. The different panels are different representations of the same set of amplitude data in $\mathrm{dB}$ SPL. The data are plotted with $f_{2} / f_{1}$ versus either (a) distortion product frequency, (b) stimulus frequency $f_{1}$, or (c) stimulus frequency $f_{2}$. In each panel, the same color-coding is used. For clarity, individual contour lines are drawn at 3-dB intervals rather than at 2-dB intervals as was used in Figure 1. For DPOAEs at $3 f_{1}-2 f_{2}$, a vertical pattern occurs when plotted as a function of $f_{1}$ (panel b). In contrast, for DPOAEs at $3 f_{2}-2 f_{1}$ patterns are only close to vertical when either primary frequency $f_{2}$ (panel c) or DPOAE frequency (panel a) is on the horizontal axis. Although the amplitudes are reduced, these results are qualitatively the same as those observed for DPOAEs at $2 f_{1}-f_{2}$ and $2 f_{2}-f_{1}$ (Fig. 1). $\pm \mathrm{SD}$ of all frogs and all frequency ratios) for DPOAEs at $2 f_{1}-f_{2}$ plotted as a function of primary frequency $f_{1}$. For $f_{1}<1250 \mathrm{~Hz}$, the group delays were larger (corresponding to more closely spaced contour lines in Figure 2) compared to the group delays for DPOAEs recorded with $f_{1}>1250 \mathrm{~Hz}$. A similar plot is given for DPOAEs at $2 f_{2}-f_{1}$ in Figure 3b. Here, the group delays are relative constant for $f_{1}>800 \mathrm{~Hz}$. Below $800 \mathrm{~Hz}$, the group delays decrease with increasing stimulus frequency.

The presence of DPOAEs was not restricted to the frequencies $2 f_{1}-f_{2}$ and $2 f_{2}-f_{1}$. In all frogs studied, DPOAEs were also detectable at $3 f_{1}-2 f_{2}$ and $3 f_{2}-$ $2 f_{1}$. However, the frequency areas in which these DPOAEs could be observed were restricted to both smaller relative (i.e., $f_{2} / f_{1}$ ) and absolute stimulus frequency ranges. This was attributable to the smaller amplitudes of these DPOAEs. Figure 4 shows amplitudes of DPOAE at $3 f_{1}-2 f_{2}$ and $3 f_{2}-2 f_{1}$ in a similar representation as Figure 1. For DPOAE amplitude at $3 f_{1}-2 f_{2}$, i.e., the upper halves of Figure 4 , the peaks and valleys do not shift in frequency with varying stimulus frequency ratio when stimulus frequency $f_{1}$ (Fig. 4b) is on the abscissa. In contrast, in the lower halves of Figure 4 , i.e., DPOAE at $3 f_{2}-2 f_{1}$, the amplitude peaks and valleys give rise to an approximately vertical pattern in the contour plots when either DPOAE frequency (Fig. 4a) or stimulus frequency $f_{2}$ (Fig. 4c) is on the horizontal axis.

In only one frog were these higher-order DPOAEs observed over a large enough stimulus frequency area

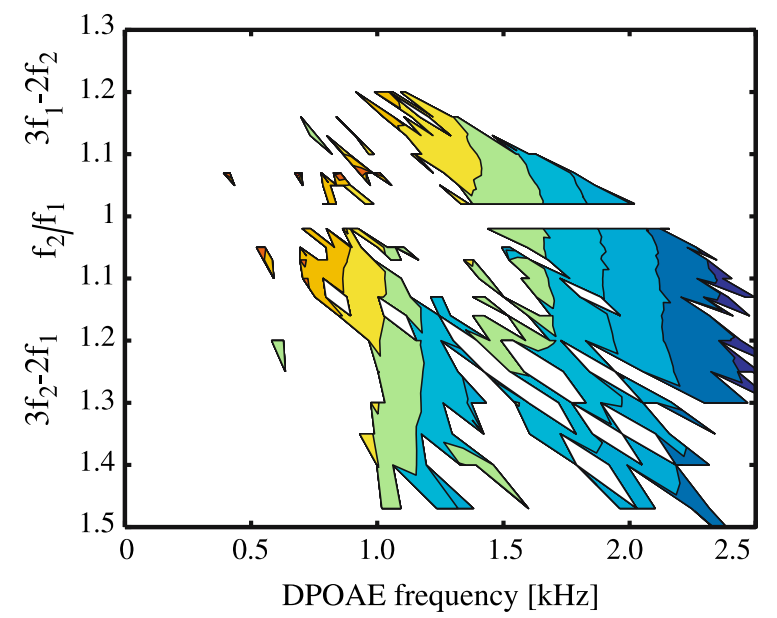

FIG. 5. Relative phase of DPOAE at $3 f_{1}-2 f_{2}$ and $3 f_{2}-2 f_{1}$ plotted as a function of distortion product frequency. Data correspond to amplitude data represented in Figure 4. Contour lines are drawn at $\pi$ rad intervals. The vertical contour lines indicate that the relative phase of the DPOAEs depends on the frequency of the distortion product alone, and is independent of both the absolute and relative frequencies of the two stimulus tones. This dependence on distortion product frequency is similar to that observed for the relative phase data for DPOAEs at $2 f_{1}-f_{2}$ and $2 f_{2}-f_{1}$ (Fig. 2). 
to reliably unwrap the phase data. The phase data obtained in this frog are shown in Figure 5. This figure corresponds to the amplitude data shown in Figure 4. For both of the higher-order DPOAEs, phase showed vertical contour lines when the DPOAE frequency is plotted on the horizontal axis (Fig. 5). These observations in both the amplitude and phase data for DPOAE at $3 f_{1}-2 f_{2}$ and $3 f_{2}-2 f_{1}$ are similar to those made for DPOAE at $2 f_{1}-f_{2}$ and $2 f_{2}-f_{1}$.

\section{DISCUSSION}

In the present study, DPOAEs were only found over a limited range of stimulus frequencies. This range is within the frequency range to which nerve fibers from the frog ear are tuned $(100-2300 \mathrm{~Hz}$ in $R$. pipiens pipiens; Ronken 1990). This frequency range could be divided in two broad frequency subregions where DPOAEs exhibited relatively large amplitudes. These subregions were separated by a frequency notch where relatively small DPOAE amplitudes were found. Based on the bandwidth of neural tuning curves recorded in R. pipiens pipiens, Ronken (1991) estimated that nerve fibers tuned to frequencies less than $1250 \mathrm{~Hz}$ innervate the amphibian papilla, while nerve fibers tuned to higher frequencies innervate the basilar papilla. The correspondence between the DPOAE data and the nerve fiber data suggests that frog DPOAEs originate from both of the papillae present in the inner ear. A similar observation was made by Van Dijk and Manley (2001), Van Dijk et al. (2003), and Meenderink and Van Dijk (2004). At the stimulus levels used here $\left(L_{1}=L_{2}=76 \mathrm{~dB}\right.$ SPL $)$, DPOAE amplitude was larger in the $\mathrm{BP}$ than in the AP for all frogs.

The phase, obtained when recording these DPOAE audiograms, varies with varying stimulus frequencies. For DPOAEs from the AP, the rate of phase change decreases for increasing frequency, resulting in the observed frequency dependence of the group delay (Fig. 3). For DPOAEs generated in the $\mathrm{BP}$, group delays are relatively constant, signifying a nearly linear change in phase with changing frequencies. This dependence of group delay on frequency (in the AP) is not only similar to the frequency dependence of response delays reported for neural data in frogs (Hillery and Narins 1984, 1987), but also to neural delays reported for all vertebrate classes (see Manley et al. 1990 for an overview). In mammals, these delays have been attributed to the delay time of the traveling wave on the basilar membrane. In fact, the close correspondence between mammalian delays and those found in the coqui frog (Hillery and Narins 1984) led to the hypothesis of a traveling wave in the tectorial membrane that covers the frog amphibian papilla. However, in the bobtail lizard no traveling wave on the basilar membrane is present (Manley et al. 1988), while the neural delays again show a similar dependence on frequency (Manley et al. 1990). It seems that the relatively similar neural delay patterns found across nonmammalian vertebrate classes arise from similarly tuned filter arrays. Thus, despite the very different auxiliary structures in the inner ears of the different vertebrate classes, filter mechanisms may be similar across species.

The patterns in DPOAE amplitude and phase described here deviate considerably from those reported for mammals (Knight and Kemp 2000; Schneider et al. 2003). In the mammalian cochlea, the patterns observed in the amplitude and accompanying phase data are oriented parallel to each other: when plotted in an $f_{2} / f_{1}$ versus $f_{d p}$ area map, both amplitude and phase display either a vertical or a horizontal orientation. A vertical orientation of the patterns occurs when the recorded DPOAE is dominated by a reflection component. This component is thought to arise via a mechanism of linear reflection occurring at irregularities of the cochlea around the characteristic place of the DPOAE (Shera and Guinan 1999). Consequently, DPOAE amplitude and phase primarily depend on the distortion product frequency, which results in the observed vertical patterns for both amplitude and phase. In contrast, a horizontal orientation of the patterns in amplitude and accompanying phase data is found if not the reflection component, but rather the nonlinear distortion component dominates the recorded DPOAE signal. This component is thought to arise via nonlinear distortion at that region along the basilar membrane where the response envelopes of the two stimulus tones overlap maximally, i.e., around the tonotopic location of primary frequency $f_{2}$. By assuming a scale-invariant cochlea with respect to frequency, the relative phase of the two stimulus tones depends only on the ratio $f_{2} / f_{1}$ and is independent of DPOAE frequency, resulting in the observed horizontal orientation of the patterns in amplitude and phase.

The close link between the patterns in DPOAE amplitude and phase, as seen in the mammalian cochlea, was not present in the frog. This is seen most clearly for lower sideband DPOAEs (with $f_{d p}<f_{1}, f_{2}$ ), i.e., DPOAEs at $2 f_{1}-f_{2}$ and $3 f_{1}-2 f_{2}$. Here, the relative phase of the DPOAEs shows a dependence on only the DPOAE frequency, i.e., vertical contour lines in the upper halves of Figures 2 and 5. However, in the corresponding area maps of the amplitude, the observed patterns are clearly diagonal (Figs. 1a and 4a, upper halves). This diagonal orientation of the patterns in amplitude data when represented in an 
$f_{2} / f_{1}$ versus $f_{d p}$ map indicates that the DPOAE amplitude does not exclusively depend on either the relative stimulus frequencies or the DPOAE frequency. Rather, the observed patterns are oriented vertically when the primary frequency $f_{1}$ is plotted on the horizontal axis (Figs. 1b and 5, upper halves).

For upper sideband DPOAE (with $f_{d p}>f_{1}, f_{2}$ ), i.e., $2 f_{2}-f_{1}$ and $3 f_{2}-2 f_{1}$, similar differences between mammalian and frog DPOAEs were present. Again, the vertical contour lines in the phase data (Figs. 2 and 5, lower halves) indicate a dependence of DPOAE phase on distortion product frequency alone. On the other hand, the patterns in amplitude data are slightly diagonal when DPOAE frequency is on the horizontal axis. A similar diagonal orientation is observed when amplitude is plotted as a function of stimulus frequency $f_{2}$ (Figs. 1 and 4 , lower halves).

Knight and Kemp (2001) describe their results, obtained in the cochlea, with a transmission line model. The model is not a physical description of the cochlea, but it incorporates some generic cochlear properties, such as the traveling wave on the basilar membrane. The difference in the amplitude and phase patterns observed in mammals and frogs indicate that the model does not apply to the frog inner ear. In other words, our results do not provide any evidence for cochlear-like traveling wave mechanics in the frog inner ear.

But if no cochlear-like traveling wave mechanics are involved in the sound transduction in the frog inner ear, what kind of mechanism is?

Of the two hearing organs present in the frog inner ear, the basilar papilla is the simplest in structure and function. It consists of a small patch of hair cells, which is embedded in a solid surface and covered by a tectorial membrane. The papilla essentially functions as a single auditory filter. Evidence for this is provided by tuning curves obtained from nerve fibers innervating this organ: in individual frogs almost all nerve fibers are tuned to a single frequency, with the shapes of all tuning curves being remarkably similar (Ronken 1990). Obviously, a traveling wave model is not applicable here.

Based on the notion that the BP functions as a single auditory filter, we will consider a single resonator, the Duffing oscillator, as a simple model for DPOAE generation in this papilla (see also Van Dijk and Manley 2001). The Duffing oscillator is described by the nonlinear second-order equation

$$
m \ddot{x}+R \dot{x}+k(x) x=F(t)
$$

where $m$ is a mass, whose movement is driven by an external force $F(t)$. The linear resistance $R$ impedes this movement, while a nonlinear stiffness $k(x)=$ $k_{0}\left(1+\frac{x^{2}}{x_{0}^{2}}\right)$ tries to restore the mass' position $x$ to its equilibrium position $x=0$.

When the oscillator is driven by a two-tone force

$$
F(t)=A_{1} \sin \left(2 \pi f_{1} t\right)+A_{2} \sin \left(2 \pi f_{2} t\right)
$$

cubic distortion products are present in the response $x(t)$. We simulated the oscillator using a range of absolute and relative stimulus frequencies: $f_{1}$ varied between 0.2 and 2.0 in 0.01 -steps, with the resonance frequency of the oscillator being 1 , while $f_{2} / f_{1}$ was between 1.04 and 1.7 in steps of 0.01 . This allowed for a qualitative comparison between the model and the DPOAEs recorded in the frog. The results of the model are shown in Figure 6 for $m=1, r=1, k_{0}=4 \pi^{2}$, $x_{0}=1$, and $A_{1}=A_{2}=1$. In this figure, the amplitude and phase data are plotted as a function of intermodulation product frequency, similar to what was performed for the DPOAEs recorded from the frog ear (Figs. 1a and 2).

The amplitude contour lines, drawn at 10-dB intervals (Fig. 6a), show a pattern that is very similar to that observed in the recorded DPOAEs (Fig. 1a). For intermodulation products at $2 f_{1}-f_{2}$ (Fig. 6a, upper half) this pattern has a diagonal orientation, following lines of fixed $f_{1}$. In contrast, for intermodulation products at $2 f_{2}-f_{1}$ (Fig. 6a, lower half) the pattern in the contour lines is approximately vertical. Notice that in both the lower and upper halves of the panel contour lines exhibit three more or less pronounced lobes. These arise whenever $f_{1}, f_{2}$ or $f_{d p}$ coincides with the resonance frequency of the oscillator.

The phase of the intermodulation products produced by the model are shown in Figure 6b. It can be seen that the phase obtained with a single fixed-ratio sweep remains largely unchanged. Only when the intermodulation product frequency varies from slightly below to slightly above the resonance frequency does phase change rapidly by $\pi \mathrm{rad}$. The contour lines in Figure $6 \mathrm{~b}$ follow lines of equal intermodulation product frequency, resulting in a vertically oriented pattern which is similar to that observed in frog DPOAE.

The qualitative good agreement between the patterns in DPOAE and intermodulation products, both in amplitude and phase, provides further support that the frog basilar papilla functions as a single (nonlinear) resonator.

DPOAEs from the amphibian papilla, the other hearing organ in the frog ear, behave similar to those from the BP: the patterns of DPOAE amplitude and phase (Figs. 1 and 2, respectively) are similar below (AP) and above (BP) the notch frequency of $1250 \mathrm{~Hz}$. Consequently, as with the BP, emissions from the AP can be described by the simple oscillator model given in Eq. (2). However, the AP is not a single auditory 
a) amplitude

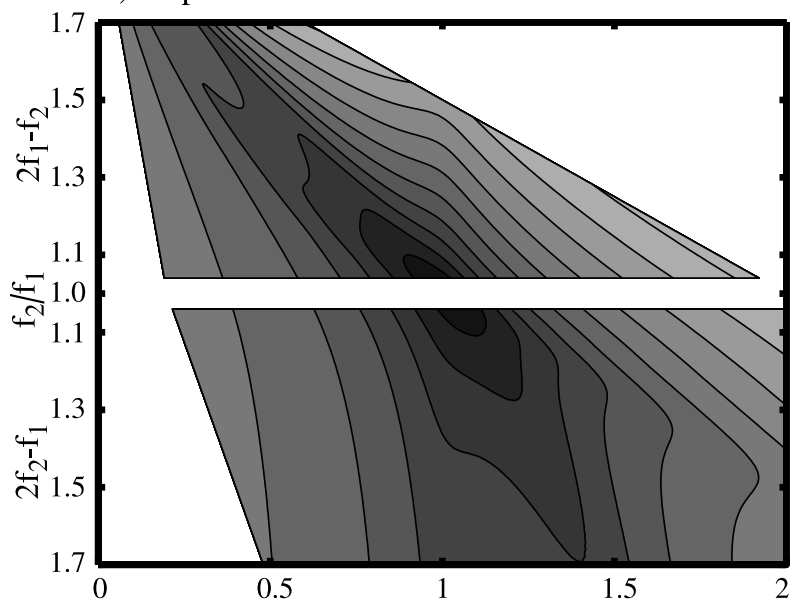

b) phase

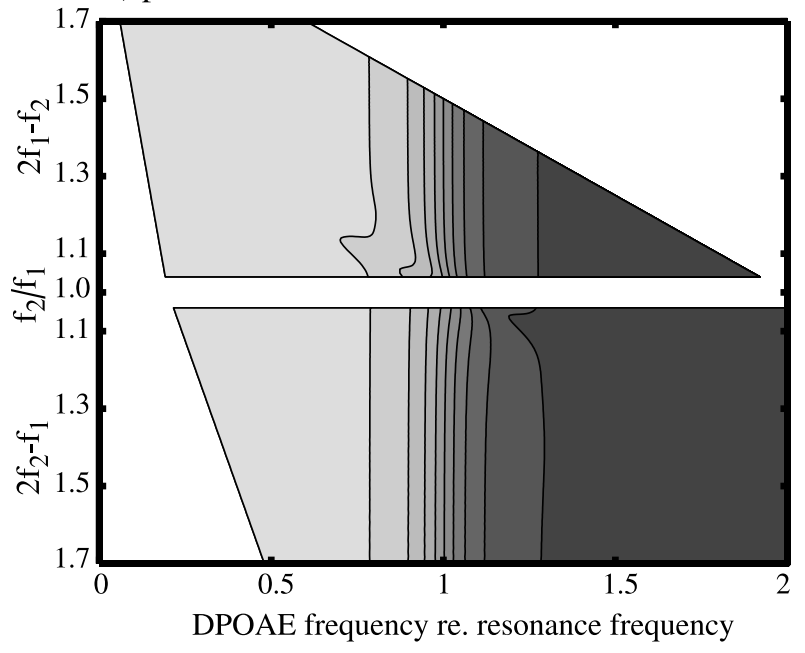

FIG. 6. Intermodulation products at $2 f_{1}-f_{2}$ and $2 f_{2}-f_{1}$ obtained from a single nonlinear resonator used to model the frog basilar papilla. The model consisted of a Duffing oscillator which was driven by a two-tone force. a) Amplitude in $\mathrm{dB}$ plotted as a function of intermodulation product frequency, similar to the presentation of DPOAE amplitude in Figure 1a. Individual contour lines are drawn at $10-\mathrm{dB}$ intervals. The pattern formed by the contour lines is very similar to that observed in the recorded DPOAE. For $2 f_{1}-f_{2}$ (upper half of the panel), the pattern is oriented diagonally, following lines of fixed $f_{1}$. In contrast, for $2 f_{2}-f_{1}$ (lower half of the panel) the pattern is closer to vertical. The individual contour lines for both intermodulation products all exhibit three more or less distinct lobes, which are not observed in the DPOAE data. These lobes occur whenever $f_{1}, f_{2}$ or $f_{d p}$ coincides with the resonance frequency of the oscillator. Apart from these lobes, the pattern of the contour lines displayed here is similar to that in Figure 1a for the basilar papilla $(>1250 \mathrm{~Hz})$. b) Phase, in rad, accompanying the amplitude data in panel a. It is plotted relative to the $f_{1}$ and $f_{2}$ stimulus frequency components in the response $x(t)$ (see Eq. (2)), rather than the force $F(t)$. This compensates for the nonlinear phase response of the model. Individual contour lines are drawn at $0.1 \pi$ rad intervals. Again, the data are plotted as a function of intermodulation product frequency. As for the DPOAE phase (Fig. 2), the contour lines are vertical, irrespective of the intermodulation frequency (i.e., $2 f_{1}-f_{2}$ or $2 f_{2}-f_{1}$ ). This indicates that the phase primarily depends on the frequency of the intermodulation product, and not on the two stimulus frequencies. filter, but functions as a tonotopically organized array of auditory filters (Lewis and Leverenz 1983). How can the simple BP-like DPOAE characteristics be reconciled with the structural and functional properties of the AP? We will discuss various factors which may contribute to the answer of this question, but at present we are unable to provide a definitive answer.

The AP is situated in a short chamber which runs from the periotic canal to the saccular recess. A contact membrane separates the perilymph in the periotic canal from the endolymph in the papillar space. The sensory hair cells are situated in the roof of the chamber, and are embedded in a rigid support structure. Hanging from the hair cells is an acellular structure, the tectorial membrane. Approximately halfway along the papilla, a tectorial curtain projects from the tectorial membrane to the floor of the papillar chamber. Because of its positioning within the chamber, any fluid movement along the principal axis of the papilla will result in movement of this tectorial curtain.

By tracing auditory nerve fibers with known characteristic frequencies to their point of innervation within the AP, Lewis et al. (1982) showed that the tonotopic organization is along the principal axis of the papilla. Here, the highest frequencies are at the caudal part of the AP (close to the contact membrane that separates the peri- and endolymph), while toward the rostral end (closer to the sacculus) the characteristic frequency of nerve fibers decreases. Although acoustic energy enters the papillar chamber from the saccular space (Purgue and Narins 2000), it seems that the excitation moves from high to low frequencies, similar to the cochlea. This is manifest in the first-spike latency for auditory nerve fibers, which is longest for low-frequency fibers and shortest for fibers tuned to the highest frequencies within in the AP (Hillery and Narins 1987). Neural tuning curves from the AP, show shallow slopes below the characteristic frequency, and steep slopes above (Narins and Hillery 1983). Therefore, Lewis and Leverenz (1983) concluded that the AP, like the cochlea, may be modeled as a transmission-line lowpass filter: each section of the papilla absorbs highfrequency acoustic energy, but passes lower frequencies to the subsequent sections in the structure. Although such a model describes the functional behavior of the papilla, Lewis and Leverenz (1983) were unable to reconcile it with the known anatomical gradients in the papilla.

They emphasized that the implementation of such a low-pass filter mechanism in the amphibian papilla must be very different from that in the cochlea. The basilar membrane in the cochlea is between two fluidfilled scalae, and is excited by a pressure difference between these scalae. The mechanical coupling 
between the different sections of the basilar membrane is provided through the fluid within the scalae: when the basilar membrane moves down at a particular location, it pushes away fluid within the scala tympani. This fluid has to push up (toward scala vestibuli) the basilar membrane at a different location in the cochlea. This fluid coupling contributes to the traveling wave propagation on the basilar membrane. Consequently, it contributes significantly to the DPOAE patterns, as observed by Knight and Kemp (2000) and Schneider et al. (2003), because these patterns reflect both forward and reverse travel of acoustic excitation along the basilar membrane.

In contrast with the cochlea, the AP is not between two fluid-filled canals. Therefore, coupling between the different sections of this papilla must be different, where some sort of coupling via the tectorial membrane is most likely to occur. As sound enters the AP from the saccular space, it moves along the principal axis of the papilla. Two modes of excitation of the tectorial membrane can be considered (Lewis and Leverenz 1983): (1) fluid flow in the recess of the AP may move the tectorial curtain that spans the recess. This excitation may travel down the rest of the tectorium and may thus stimulate the entire papilla. Alternatively, (2) frictional coupling between fluid flow and the tectorial membrane may excite the papilla along its entire length. Because both the tectorial membrane mechanics and the coupling between subsequent sections in the papilla are different from that in the cochlea, it is not surprising that the observed DPOAE patterns (this work) differ from those in the cochlea (Knight and Kemp 2000; Schneider et al. 2003). However, at present, it is impossible to decide what mechanism describes the observed behavior in the AP, as no data are available on the mechanics of the tectorial membrane.

It is important to note that the differences between the amphibian and the mammalian DPOAEs need not reflect a difference in the emission generation mechanism itself. In frogs, the dependence of DPOAE amplitude on stimulus levels is similar to that in mammals (Meenderink and Van Dijk 2004). This strongly suggests important similarities between DPOAE generation in frogs and mammals. Note that the broad similarities across vertebrate species also include similar neural delays (reviewed in Manley et al. 1990) and similar frequency selectivity in neural tuning (reviewed in Manley 1990).

In conclusion, we showed that the dependence of DPOAE amplitude and phase on the stimulus frequencies is conspicuously different from that in mammals. Our DPOAE measurements did not give any evidence for a mammalian-like traveling wave in the frog inner ear. For the frog's basilar papilla, this is not surprising and our results are consistent with the view that this papilla essentially functions as a single auditory filter. For the amphibian papilla, a traveling wave on the tectorial membrane has been hypothesized in the past. This hypothesis is not supported by our results. The differences described here need not reflect fundamental differences between DPOAE generation mechanisms, but presumably reflect the different mechanical properties of the auxiliary structures between the amphibian and basilar papilla and the mammalian cochlea.

\section{ACKNOWLEDGMENTS}

This work was supported by grants from the Netherlands Organization for Scientific Research (NWO), and the Heinsius Houbolt Foundation to S.W.F.M. and P.v.D., and NIH grant DC-00222 to P.M.N.

\section{REFERENCES}

Brown AM, Harris FP, Beveridge HA. Two sources of acoustic distortion products from the human cochlea. J. Acoust. Soc. Am. 100:3260-3267, 1996.

FRISHKOPF LS, FLOCK A. Ultrastructure of the basilar papilla, an auditory organ in the bullfrog. Acta Otolaryngol. 77:176-184, 1974.

Hillery CM, NARINS PM. Neurophysiological evidence for a traveling wave in the amphibian inner ear. Science 225: 1037-1039, 1984.

Hillery CM, Narins PM. Frequency and time domain comparison of low-frequency auditory fiber responses in two anuran amphibians. Hear. Res. 25:233-248, 1987.

Kemp DT, Brown AM. An integrated view of cochlear mechanical nonlinearities observable from the ear canal. In: de Boer E, Viergever MA (eds) Mechanics of Hearing. Delft University Press, Delft, pp 75-82, 1983.

KIM DO. Cochlear mechanics: implications of electrophysiological and acoustical observations. Hear. Res. 2:297-317, 1980.

KNIGHT RD, Kemp DT. Indications of different distortion product otoacoustic emission mechanisms from a detailed $f_{1}, f_{2}$ area study. J. Acoust. Soc. Am. 107:457-473, 2000.

KNight RD, Kemp DT. Wave and place fixed DPOAE maps of the human ear. J. Acoust. Soc. Am. 109:1513-1525, 2001.

LEwIS ER. Comparative studies of the anuran auditory papillae. Scan. Electron Microsc. II:633-642, 1978.

LEwIs ER. On the frog amphibian papilla. Scan. Electron Microsc. IV:1899-1913, 1984.

LEWIS ER. Convergence of design in vertebrate acoustic sensors. In: Webster DB, Fay RR, Popper AN (eds) The Evolutionary Biology of Hearing. Springer-Verlag, New York, pp 163-184, 1992.

Lewis ER, Leverenz EL. Morphological basis for tonotopy in the anuran amphibian papilla. Scan. Electron Microsc. I:189-200, 1983.

LewIS ER, NARINS PM. The acoustic periphery of amphibians. In: Fay RR, Popper RR (eds) Comparative Hearing: Fish and Amphibians. Springer-Verlag, New York, pp 101-154, 1999.

Lewis ER, Leverenz EL, Koyama H. The tonotopic organization of the bullfrog amphibian papilla, an auditory organ lacking a basilar membrane. J. Comp. Physiol. 145:437-445, 1982. 
Manley GA. Peripheral Hearing Mechanisms in Reptiles and Birds. Springer-Verlag, Berlin, 1990.

Manley GA, Yates GK, Köppl C. Auditory peripheral tuning: evidence for a simple resonance phenomenon in the lizard Tiliqua. Hear. Res. 33:181-190, 1988.

Manley GA, Yates GK, Köppl C, Johnstone BM. Peripheral auditory processing in the bobtail lizard Tiliqua rugosa. IV. Phase locking of auditory-nerve fibres. J. Comp. Physiol. A 167:129-138, 1990.

Meenderink SWF, VAN DIJK P. Level dependence of distortion product otoacoustic emissions in the leopard frog, Rana pipiens pipiens. Hear. Res. 192:107-118, 2004.

NARINS PM, Hillery CM. Frequency coding in the inner ear of anuran amphibians. In: Klinke R, Hartmann R (eds) HearingPhysiological Bases and Psychophysics. Springer-Verlag, Heidelberg, pp 70-76, 1983.

Purgue AP, Narins PM. Mechanics of the inner ear of the bullfrog (Rana catesbeiana): the contact membranes and the periotic canal. J. Comp. Physiol. A 186:481-488, 2000.

Robbins RG, Bauknight BS, Honrubia MD. Anatomical distribution of the efferent fibers in the VIIIth cranial nerve of the bullfrog Rana catesbeiana. Acta Otolaryngol. 64:436-448, 1967.

RONKEN DA. Basic properties of auditory-nerve responses from a 'simple' ear: the basilar papilla of the frog. Hear. Res. 47:63-82, 1990.
RonkEn DA. Spike discharge properties that are related to the characteristic frequency of single units in the frog auditory nerve. J. Acoust. Soc. Am. 90:2428-2440, 1991.

Schneider S, Prijs VF, Schoonhoven R. Amplitude and phase of distortion product otoacoustic emissions in the guinea pig in an $\left(f_{1}, f_{2}\right)$ area study. J. Acoust. Soc. Am. 113:3285-3296, 2003.

Shera CA, Guinan JJ. Evoked otoacoustic emissions arise by two fundamentally different mechanisms: a taxonomy for mammalian OAEs. J. Acoust. Soc. Am. 105:782-798, 1999.

Stover LJ, Neely ST, Gorga MP. Latency and multiple sources of distortion product otoacoustic emissions. J. Acoust. Soc. Am. 99:1016-1024, 1996.

Talmadge CL, Long GR, Tubis A, Dhar S. Experimental confirmation of the two-source interference model for the fine structure of distortion product otoacoustic emissions. J. Acoust. Soc. Am. 105:275-292, 1999.

VAN DijK P, Manley GA. Distortion product otoacoustic emissions in the tree frog Hyla cinerea. Hear. Res. 153:14-22, 2001.

Van Dijk P, Wit HP, Segenhout JM. Dissecting the frog inner ear with Gaussian noise. I. Application of high-order Wiener-kernel analysis. Hear. Res. 114:229-242, 1997.

Van Dijk P, Narins PM, Mason MJ. Physiological vulnerability of distortion product otoacoustic emissions from the amphibian ear. J. Acoust. Soc. Am. 114:2044-2048, 2003. 